\title{
MULTI-AGENT SYSTEM FOR SIMULATION OF SURROUNDING THE SEA VESSEL
}

\author{
Petr Sosnin \\ Computer Department, \\ Ulyanovsk State Technical University, \\ Severny Venetc, Ulyanovsk, 432027, Russia \\ E-mail: sosnin@ulstu.ru
}

\section{KEYWORDS}

Multi-agent system, radar station, sea vessel.

\begin{abstract}
There are many different tasks which are to be solved on a sea vessel on the base of the primary information from radar stations (RSs) about moving objects in the vessel surrounding. Frequently enough such information is being entered from several RSs with different characteristics. The redundancy of informational flows is used for achieving many positive effects, for example for increasing the adequacy of vessel environment models. The processing of such redundant information is named as data fusion. The article presents the multi-agent system (MAS) for solving the data fusion task on the sea vessel. The suggested MAS uses the program agents not only for dynamic objects but for units of informational flows as well.
\end{abstract}

\section{INTRODUCTION}

The rationality of the sea vessel navigation essentially depends on adequate monitoring the above-water and air environment. In the general case such environment includes different kinds of moving the water and air objects with different characteristics and some of them can be combined in groups (Waard 2008).

The different kinds of vessels navigation equipment and other outside navigation systems are appointed to gathering the necessary information for solving the data fusion task. All of these means are combined in a multisensor multi-target tracking system (MTTS) of the vessel. Such MTTS is very important for the warship.

Let us mark that "Data fusion is now the recognized term for the processing needed to convert the data into an up-to-date model (or picture) of the world-ofinterest, suitable for human decision making ((Waard 2008)."

In this article the multi-agent approach to solving the data fusion task in the frame of the MMTS is presented. The solution is aimed at the simulation of the targets picture around the vessel $\mathrm{V}_{0}$ of the warship type.
First of all agents are used for modeling the targets each of which can be a vessel or an air object. But agents also are used for presenting features (characteristics) of discovered targets.

The suggestion takes into account that several radar stations (RS) are used on the vessel as sources of primary informational flows about observing targets. Moreover, different RSs can have different time of observing (scan time $\mathrm{T}^{\mathrm{x}}$ ) and can generate the information with different types of targets characteristics.

The use of agents simplifies the solution of the data fusion task in described conditions. The agent approach to the simulation of the vessels movement is applied to the collision avoidance task as well. In this task the agent model of the own vessel $\mathrm{V}_{0}$ is created and combined with agents of other vessels moving inside the investigating area.

Requirements of suggestion means are defined for their implementation in the Question-Answer Instrumental System WIQA (Sosnin 2009). That helps to adjust and unite the tasks of identifying targets and their groups with tasks of visualizing the targets picture around the vessel $\mathrm{V}_{0}$ and with the collision avoidance task of vessels. The model of the international rules for vessels collision avoidance was created and included into the developed multi-agent system named MasWIQA.

\section{RELATED WORKS}

The data fusion task can be divided on subtasks by different ways. But in any case the division will include the subtask of normalizing data flows from different sensors, the subtask of the data association, the subtask of targets grouping (on the sea surface and in the airspace) and the visualization task which simulates the targets picture around the vessel $\mathrm{V}_{0}$. All of these basic subtasks are solving in MasWIQA with the help of a set of tasks which are being solved by software agents. Moreover, the special means are included to MasWIQA for solving the collision avoidance task for vessels on the base of data obtained from the data fusion task.

These positions were taken into account for analyzing the related works. The analyses has helped to define three important types of related works connected with 
the data association, collision avoidance and the usage of software agents in named tasks and subtasks.

For the data association task it is possible to indicate the following algorithmic versions:

1. Algorithms based on statistical properties of estimations of coordinates and parameters of the targets, namely, algorithms existential association (Capponi et al. 2004; Kaplan et al. 2008).

2. Algorithms of stochastic checking of data in a combination to multi-alternative algorithms of tracking (Puranik and Tugnait 2007).

3. Algorithms based on the usage of additional sources of the information (Bar-Shalom et al. 2005).

4. Algorithms of the data association based on the usage of Neural Networks (Chung et al. 2007).

As a rule, for the majority of the marked algorithms (and related them) the association is fulfilled on two stages. The first association stage consists of integrating the corresponding data values in current moments of the modeling time. At the second stage the received groups of data are being identified with already available trajectories of targets, on the base of various properties of the received data.

It is necessary to notice that the Internet- search of publications about data fusion with multi-agent solutions has been without interesting results.

For decreasing the risks of collisions, the international rules for avoidance (COLREG-72) are agreed for their execution by vessels of various types (Cockcroft 2003). In general case the vessel collision avoidance is a complicated dynamic process the exact theoretical description of which is impossible even for two moving ships. Therefore such processes are being fulfilled on the base of precedents, simple models of situations and decisions of the responsible persons.

To improve the safety of vessels the different kinds of expert systems have been created, for example in the paper (Grabowski and Wallace 1993) on the base of gaming, in the paper (Tran et al. 2000) on the base of journey planning and in the paper (Liu et al. 2006) on the base of Fuzzy-Neural Network possibilities. It is necessary to notice that in all named papers normative rules of the COLREG-72 is not mentioned.

The expert system described in this paper uses the multi-agent technique and modeling the reasoning. The possibilities of multi-agent modeling the vessels are presented in the paper (Liu and Yang 2007) where agents are used for simulation aims but without direct access to COLREG rules. The principal role of casebased reasoning in the collision avoidance task is presented in the paper (Liu et al. 2008). But combining the multi-agent analysis with question-answer reasoning is not used in related papers.

\section{MODEL OF INFORMATIONAL FLOWS}

In the developed simulation system as in related systems the first step of the data fusion process begins with solving the subtask of normalizing data flows from different sensors. The solution of this subtask is based on the following mathematical model of a set of informational flows:

$$
\begin{aligned}
& \left.\mathrm{G}=\mathrm{G}^{\mathrm{P}} \cup \mathrm{G}^{\mathrm{S}}=\left\{\mathrm{G}_{\mathrm{k}}^{\mathrm{p}}\right\} \cup\left\{\mathrm{G}_{\mathrm{k}}^{\mathrm{s}}\right\}=\left\{\left\{\mathrm{g}_{\mathrm{kr}}^{\mathrm{p}}\left(\mathrm{t}_{\mathrm{i}, \mathrm{j}}\right)\right\}\right\} \cup\left\{\mathrm{g}_{\mathrm{kr}}^{\mathrm{s}}\left(\mathrm{t}_{\mathrm{i}, \mathrm{j}}\right)\right\}\right\}, \\
& \mathrm{g}^{\mathrm{p}}{ }_{\mathrm{kr}}\left(\mathrm{t}_{\mathrm{i}, \mathrm{j}+1}\right)=\mathrm{g}_{\mathrm{kr}}^{\mathrm{p}}\left(\mathrm{t}_{\mathrm{i}, \mathrm{j}}\right)+\Delta \mathrm{g}_{\mathrm{kr}}^{\mathrm{p}}\left(\mathrm{t}_{\mathrm{i}, \mathrm{j}+1}\right) \text {, } \\
& \left.\Delta \mathrm{g}^{\mathrm{p}}{ }_{\mathrm{kr}}\left(\mathrm{t}_{\mathrm{i}, \mathrm{j}+1}\right)=\mathrm{f}_{\mathrm{r}}\left(\mathrm{G}_{\mathrm{k}}^{\mathrm{p}}\left(\mathrm{t}_{\mathrm{ij}}{ }^{\prime}\right), \mathrm{G}^{\mathrm{C}}\left(\mathrm{t}_{\mathrm{ij}}{ }^{\prime}\right), \mathrm{t}_{\mathrm{ij}}, \Delta \mathrm{t}_{\mathrm{ij}}\right)\right) \text {, } \\
& \left.\mathrm{F}=\left\{\mathrm{f}_{\mathrm{r}}\left(\mathrm{G}_{\mathrm{k}}^{\mathrm{p}}\left(\mathrm{t}^{\prime}\right), \mathrm{G}^{\mathrm{C}}\left(\mathrm{t}^{\prime}\right), \mathrm{t}_{\mathrm{ij}}, \Delta \mathrm{t}_{\mathrm{ij}}\right)\right)\right\}, \\
& \mathrm{g}_{\mathrm{k}}^{\mathrm{s}}\left(\mathrm{t}_{\mathrm{i}, \mathrm{j}+1}\right)=\left\{\begin{array}{l}
\mathrm{g}_{\mathrm{k}}^{\mathrm{s}}\left(\mathrm{t}_{\mathrm{i}, \mathrm{j}}\right), \\
\mathrm{a}_{\mathrm{k}}^{\mathrm{s}}\left(\mathrm{t}_{\mathrm{i}, \mathrm{j}+1}\right), \operatorname{New}\left(\mathrm{g}_{\mathrm{k}}^{\mathrm{s}}\left(\mathrm{t}_{\mathrm{i}, \mathrm{j}+1}\right)\right),
\end{array}\right. \\
& \mathrm{t}_{\mathrm{i}, \mathrm{j}}=\mathrm{t}_{0}+\mathrm{i}^{*}\left(\mathrm{~J}^{\mathrm{p}}+1\right) * \mathrm{~T}^{\mathrm{M}}+\mathrm{j}^{*} \mathrm{~T}^{\mathrm{M}} \text {, } \\
& \mathrm{j}=1, \mathrm{~J}^{\mathrm{p}}+1, \mathrm{i}=1, \mathrm{I}, \mathrm{k}=1, \mathrm{~K}, \mathrm{p}=1, \mathrm{P}, \mathrm{r}=1, \mathrm{R},
\end{aligned}
$$

This incremental model transforms each real informational flow from the corresponding sensor $\mathrm{RS}^{\mathrm{p}}$ (standing on the vessel $\mathrm{V}_{0}$ ) to its version reflected to the modeling time with the scan time $\mathrm{T}^{\mathrm{M}}$ satisfying to a condition $\mathrm{T}^{\mathrm{M}} \leq \min \left\{\mathrm{T}^{\mathrm{p}}\right\}$. Such reflection provides the normalization of informational flows in time.

One more way of the normalizing the data flows is bound with the choice of a normative set of targets characteristics $\left\{\mathrm{G}_{\mathrm{k}}^{\mathrm{p}}\right\}=\left\{\left\{\mathrm{g}_{\mathrm{kr}}^{\mathrm{p}}\left(\mathrm{t}_{\mathrm{i}, \mathrm{j}}\right)\right\}\right\}$ obtained from the set $\left\{\mathrm{RS}^{\mathrm{p}}\right\}$ and a set of $\left\{\mathrm{G}_{\mathrm{k}}^{\mathrm{s}}\right\}=\left\{\left\{\mathrm{g}_{\mathrm{kr}}^{\mathrm{s}}\left(\mathrm{t}_{\mathrm{i}, \mathrm{j}}\right)\right\}\right\}$ obtained from other sources $\mathrm{S}^{\mathrm{s}}$ of data $\left\{\mathrm{G}_{\mathrm{k}}^{\mathrm{s}}\right\}$.

For characteristics $\left\{\left\{\mathrm{g}_{\mathrm{kr}}^{\mathrm{p}}\left(\mathrm{t}_{\mathrm{i}, \mathrm{j}}\right)\right\}\right\}$ their values are computing with the help of extrapolation $\Delta \mathrm{g}_{\mathrm{kr}}^{\mathrm{p}}\left(\mathrm{t}_{\mathrm{i}, \mathrm{j}+1}\right)$ of the corresponding scan times $\mathrm{T}^{\mathrm{p}}$ for intermediate points of time $t_{i, j}$. A set of characteristics $G^{C}\left(t_{i j}{ }^{\prime}\right)$ for the vessel $\mathrm{V}_{0}$ is used in such computing.

Any characteristic $g_{k}^{s}\left(t_{i, j+1}\right)$ in the next points of time $t_{i, j+1}$ can change the value if the definite event $\operatorname{New}\left(\mathrm{g}_{\mathrm{k}}^{\mathrm{s}}\left(\mathrm{t}_{\mathrm{i}, \mathrm{j}+1}\right)\right.$ is happened. The role of other elements of the model can be understood without explaining.

It is necessary to notice that the presented model opens possibilities for the parallel processing informational flows and such possibilities are used in their multi-agent version.

\section{MULTI-AGENT MODELING THE DATA FUSION}

The mathematical model of the set of informational flows helps to single out the flow for each characteristic of any target. It, in turn, allows to start the process of the informational redundancy for each target characteristic, for example, such as the bearing or distance each of which has a probabilistic type and is measured with definite accuracy.

The next step in processing of data is connected with solving the data association task and task of targets grouping. Each of these tasks must be solved for vessels and air objects differentially. The last data fusion process provides gathering the values of characteristics into the navigational model for each 
observed target for following use in decision-making on the vessel $\mathrm{V}_{0}$. The described chain

of actions has led the author to its multi-agent structure presented in Fig. 1.

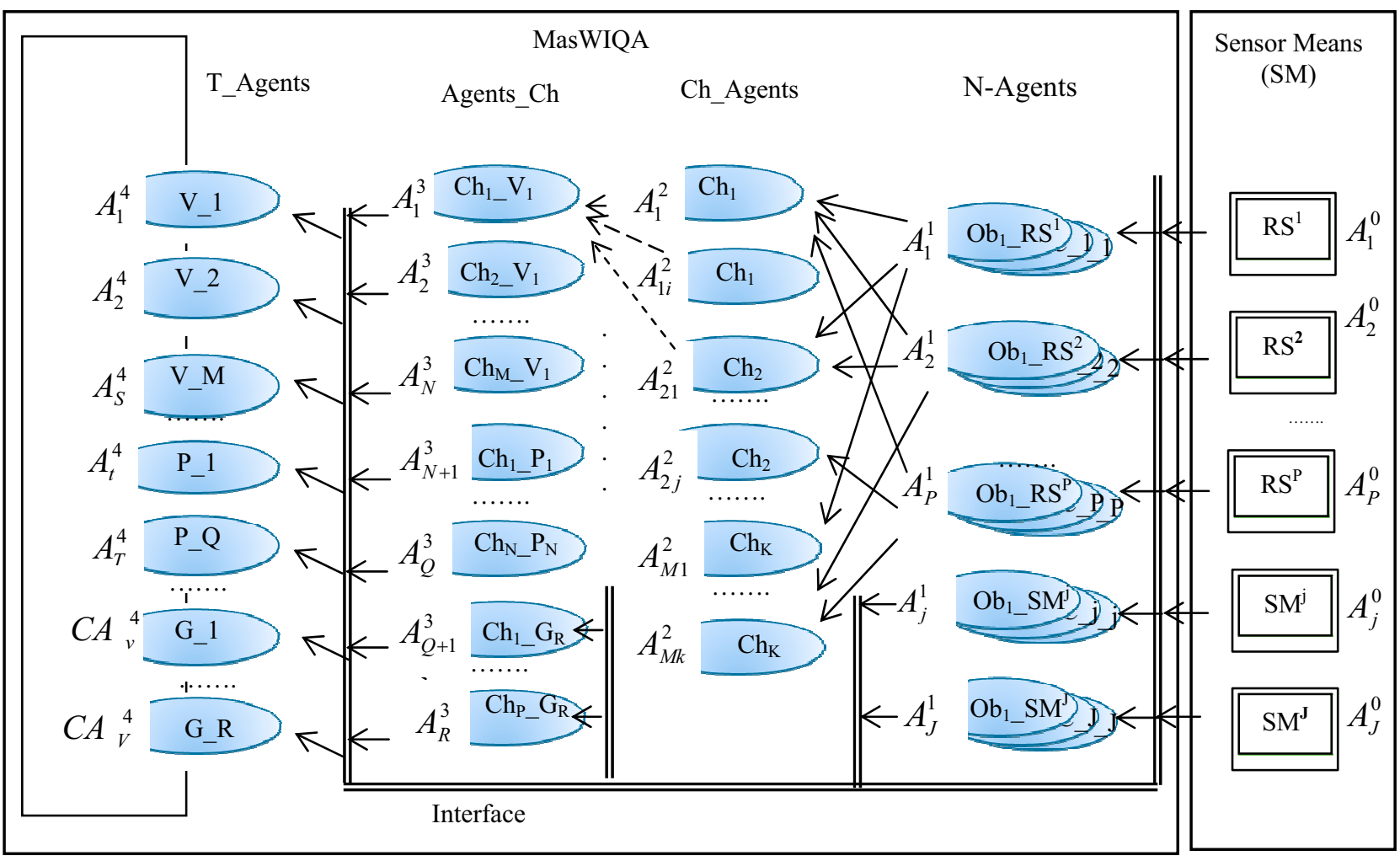

Figure 1.The multi-agent structure of data fusion processing

The following responsibilities are appointed to classes of agents:

- class $A^{0}$ of "agents" represents sensors means (SM), in particular, radar stations $\mathrm{RS}^{\mathrm{p}}$ which send flows of the primary information to MasWIQA ("agents" of this class is out the range of MasWIQA);

- $\quad$ class $A^{l}$ named "N_Agents" provides normalizing data flows from different sensors;

- class $A^{2}$ named "Ch-agents" each of which processes the redundant information about the definite characteristic from its normalized flows;

- class $A^{3}$ named "Agents-Ch" solves the data association task and the task of grouping the targets;

- class $A^{4}$ named "T_Agents" the agents of which assemble data for targets such as vessels $\left\{\mathrm{V}_{\mathrm{m}}\right\}$, air objects $\left\{\mathrm{P}_{\mathrm{q}}\right\}$ and their groups $\left\{\mathrm{G}_{\mathrm{r}}\right\}$.

The structure of MasWIQA as a whole describes by the following symbolic expression:

MasWIQA = (WIQA,Env(t), S( $\left.\left.\left\{\mathrm{A}^{1}, \mathrm{~A}^{2}, \mathrm{~A}^{3}, \mathrm{~A}^{4}\right\}\right), \mathrm{t}\right)$

$\operatorname{Env}(\mathrm{t})=(\operatorname{Cart}(\mathrm{t}), \operatorname{Aer}(\mathrm{t}), \operatorname{Isom}(\mathrm{t}))$

$A^{1}=\left\{A_{j}^{1}\left(A_{j}^{0}\right)\right\}, j=\overline{1, J}$,

$A^{2}=\left\{A_{m j}^{2}\left(S\left(A_{r}^{3},\left\{A_{j}^{1}\right\}\right)\right)\right\}, m=\overline{1, M}, j=\overline{1, J^{\prime}}, r=\overline{1, R}$,

$A^{3}=\left\{A_{r}^{3}\left(S\left(A_{v}^{4},\left\{A_{m i}^{2}\right\}\right)\right)\right\}, r=\overline{1, R}, v=\overline{1, V}, m=\overline{1, M}$,

$A^{4}=\left\{A_{v}^{4}\left(S\left(\left\{A_{r}^{3}\right\}\right)\right)\right\}, v=\overline{1, V}, r=\overline{1, R}$.
All agents which are existing in MasWIQA in definite modeling time $t$, are implemented with the usage of WIQA means added by plug-ins visualizing and simulating the targets picture as the views composition of an environment $\operatorname{Env}(t)$ around the vessel $V_{0}$. The targets picture consists of three views one of which is view Chart(t) presented in Fig. 2. It shows all vessels on the map of the definite part of the sea (the cartesian system of coordinates).

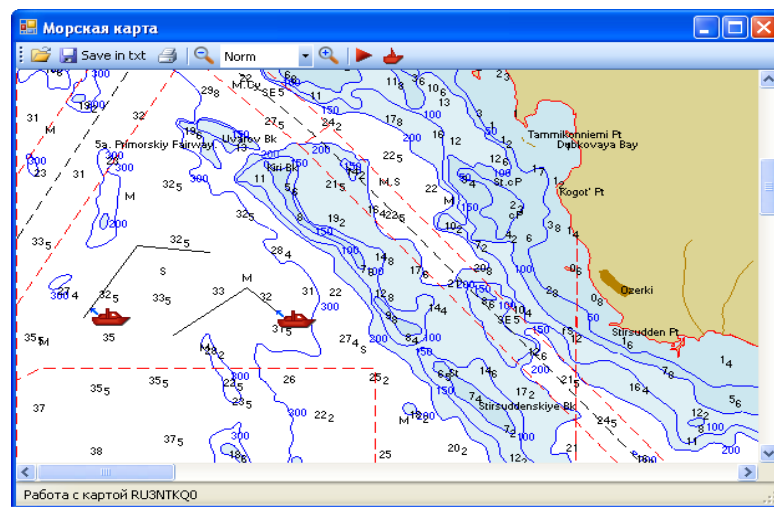

Figure 2. Map view with icons of vessels

Two other views are shown in Fig. 3 where the view Air(t) registers all air objects as usually they are viewed on the RS screen and the view Isom(t) which presents all targets in an isometric picture. 


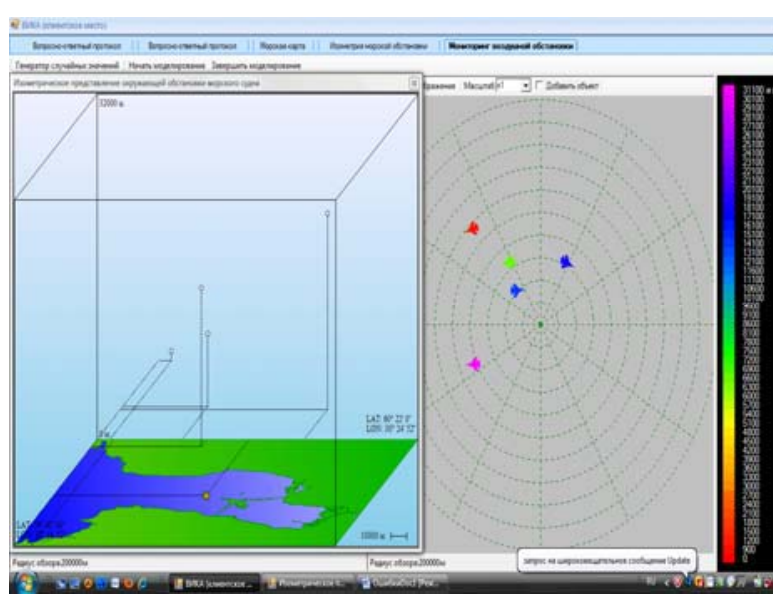

Figure 3. Model of air targets and isometric view

\section{QUESTION-ANSWER PROCESSOR WIQA}

The toolkit WIQA (Working In Questions and Answers) is intended for the maintenance of questionanswer reasoning (QA-reasoning) in the collective problem-solving and decision-making in the designing of software intensive systems. This toolkit helps to create and use the QA-model for any task including its subtasks during the conceptual solving this task. Any QA-model is developing during solving process and its finishing state is a pseudocode program of the corresponding task.

WIQA has been implemented in several versions. Its last versions named WIQA.Net, has been created on C\# at the platform of Microsoft.NET 3.5. This version of WIQA gives the possibility to execute any pseudocode program created with its help and for this reason WIQA.net can be named as the processor (QA-processor).

The main interface form of WIQA is presented in Fig. 4. This form gives the visual and touchable access to the tasks tree of the conceptual design and the QAmodel of any task of this tree.

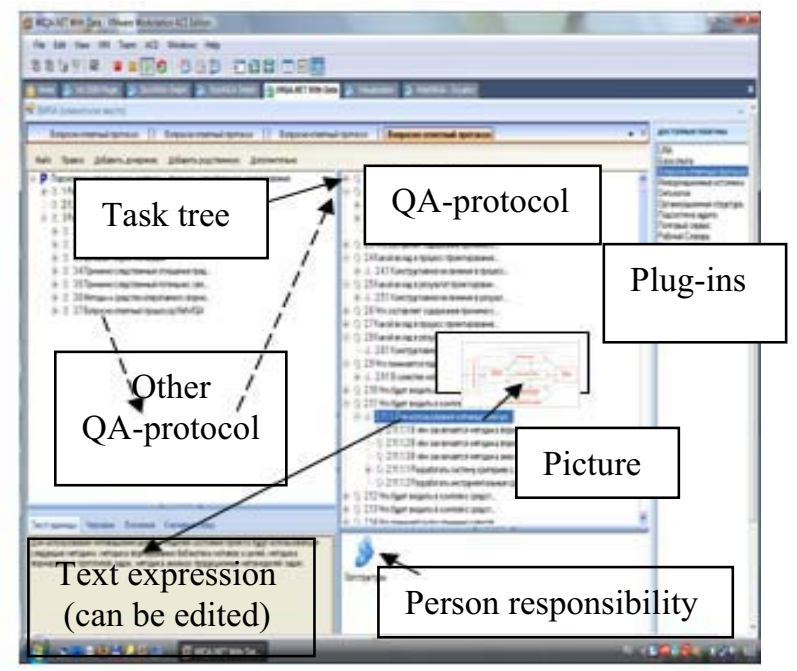

Figure 4. The main interface of WIQA
The structure of WIQA, its functional possibilities and positive effects are described in a set of publications among which the sufficient full description is given in the article (Sosnin 2009).

The features of WIQA are reflected by its general components structure presented in Fig 5. A set of specialized plug-ins have been developed in the creation of MasWIQA.

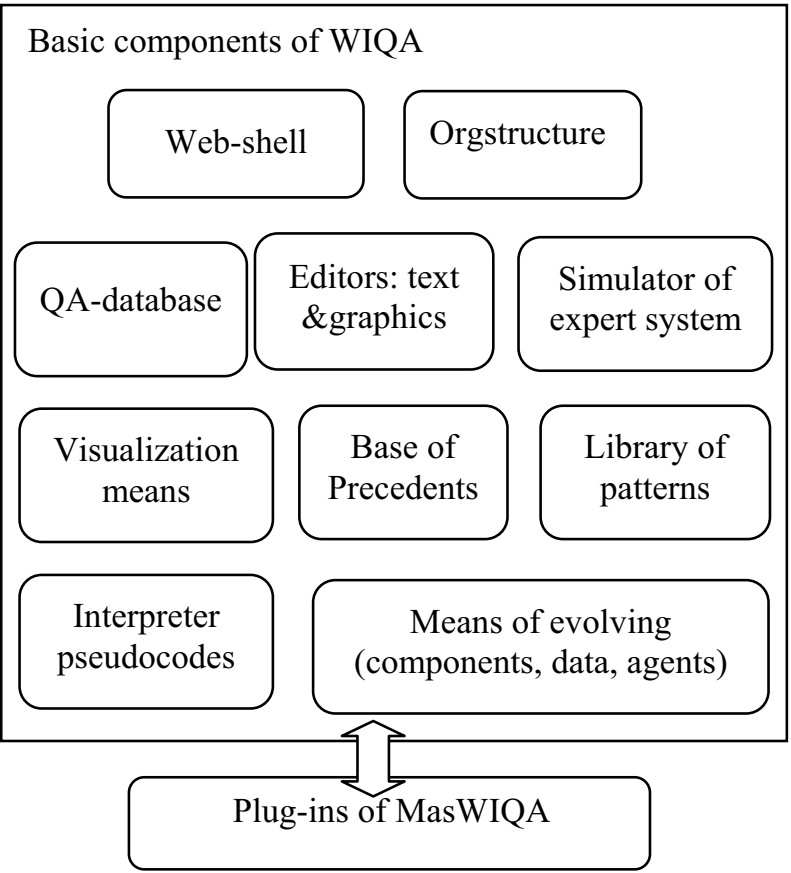

Figure 5. Components structure of WIQA

It is necessary to notice that any unit of the tasks tree and QA-model (visualized as the QA-protocol) is kept in the QA-database localized in the server of WIQA. Any such unit can be used for emulating the data of the necessary type. This possibility uses for coding the variables and their values in the form which was named as QA-data.

Events and data for software agents of MasWIQA are existed in the form of QA-data (Fig. 6)) which are registered in the announcement board and in the agents tasks included to the tasks tree of MasWIQA. Any task of the agent existence includes subordinated subtasks which use QA-data as well.

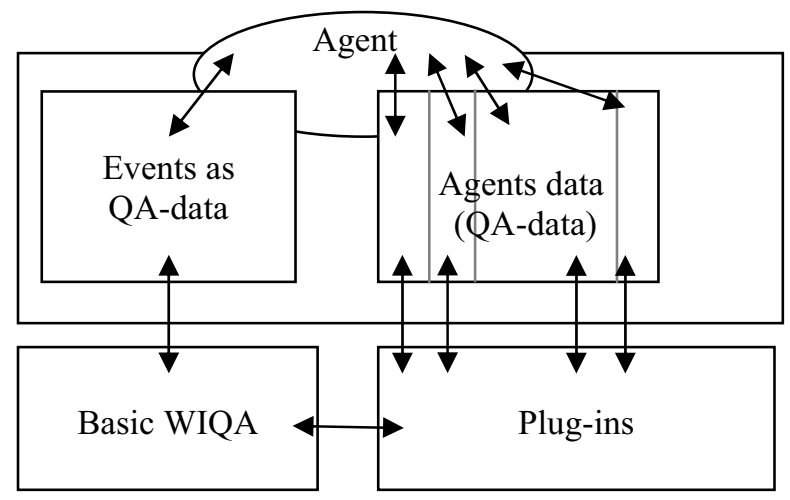

Figure 6. Connection of agent with its surrounding 


\section{DATA ASSOCIATION TASK}

After processing the entrance information by agents which are provided the normalization of informational flows, the data association subtask must be solved. This subtask can describe by the following statement:

"The set of normative flows of targets
characteristics values presents the processed
primary information about the definite set of targets
each of which could be named variously in targets
lists of different RSs. It is required to appoint the
unique name for the each target in all normalized
informational flows corresponding to this target (if
target exists in the vessel surrounding)."

This task is solved for vessels and planes a little variously but with the usage of the techniques based on the statistical hypothesis testing in both cases. The essence of the decision we shall explain on an example for two RSs which are interacting with the definite quantity of targets.

In case when $\mathrm{N}$ vessels are moving inside the investigated area the first $\mathrm{RS}^{1}$ can register $\mathrm{N}^{1}$ targets and the second $\mathrm{RS}^{2}$ can register $\mathrm{N}^{2}$ targets. It is possible that $\mathrm{N} \neq \mathrm{N}^{1} \neq \mathrm{N}^{2}$ and even $\mathrm{N}^{\mathrm{x}}>\mathrm{N}$. For each pair of targets $\mathrm{Ob}_{\mathrm{i}}$ and $\mathrm{Ob}_{\mathrm{j}}$ from targets lists of both RSs let's create two probabilistic variables (with the normal distribution)

$$
\begin{aligned}
& \xi^{\alpha}=b^{\alpha}{ }_{i}-b^{\alpha}{ }_{j} \\
& \xi^{\beta}=d^{\beta}{ }_{i}-d^{\beta}{ }_{j}
\end{aligned}
$$

where $b^{\alpha}{ }_{x}$ and $d^{\beta}{ }_{y}$ are the registering values of the bearing and distance for targets correspondingly.

If objects $\mathrm{Ob}_{\mathrm{i}}$ and $\mathrm{Ob}_{\mathrm{j}}$ represent the same object then $M \xi^{\alpha}=0, M \xi^{d}=0$ where $\mathrm{M}$ is mean (hypothesis $\mathrm{H} 0$ ); otherwise (hypothesis H1) even one of the mentioned population means should be distinct from zero.

As random variables $\xi^{\alpha}$ and $\xi^{\beta}$ are considered independent the probability of a correct identification is defined by the following expression:

$$
P_{0}=P_{0}^{(1)} \times P_{0}^{(2)},
$$

where $P_{0}^{(1)}$ - probability of a correct identification of the bearing, and $P_{0}^{(2)}-$ probability of a correct identification of the distance.

In presented case for estimations of samples the following critical values are used:

$$
\begin{aligned}
& \gamma_{\alpha}=\sigma_{\alpha} \Phi^{-1}(\sqrt{1-\varepsilon}) \\
& \gamma_{d}=\sigma_{d} \Phi^{-1}(\sqrt{1-\varepsilon})
\end{aligned}
$$

On the base of such estimations a group of software agents belonged to class $\mathrm{A}^{3}$ was created. These agents take into account all pairs of targets. They use statistics of samples and solve the appointment task by the Hungarian method.

It is necessary to notice that the data association task for air targets is solved by the similar way. In this case three random variables $\xi^{\alpha}, \xi^{\beta}$ and $\xi^{\gamma}$ are took into account. The third variable between targets heiglets.

In MasWIQA the task of targets grouping is solving by software agents as well. This task is defined as a task of image recognition the statement of which is oriented on the proximity of targets in their dynamic characteristics. The task of grouping is solving for vessels and air targets differentially.

\section{SIMULATION OF SURROUNDING}

The last subtask in data fusion is the targets visualization task. The main aim of this task is to present the targets picture in forms which are suitable for the decision-making.

A very important measure of the suitability is the accessible possibilities for estimations of the targets picture by persons who are responsible for decisions. For this reasons in all views of the targets picture the screenshots of which are presented above the distance measures are opened for their visual applying (without the use of computing).

The possibilities of estimating for air targets are supported by the use of the model of the radar screen which is added by the column of air echelons of the flight. MasWIQA opens such possibilities not only for RSs operators but for sailors on duty as well.

MasWIQA is developed as the training system. Therefore it gives the possibility for initial creating the targets picture including vessels and air targets and for appointing them random values of characteristics. The simulation process can be activated after the initial assigning.

The simulation of the targets moving is also used for solving the collision avoidance task for vessels. This task is being solved in the training regime with the usage of the specialized Expert System (ES) which is emulated by means of MasWIQA.

The simulation is aimed at the estimation of the collision threat for own vessel $\mathrm{V}_{0}$ with others moving vessels. The estimation includes two line of the behavior for the sailor on duty and for agents modeling vessels.

The sailor on duty must act in accordance with COLREG' 72 but this document is a very complicated. Therefore all rules of COLREG'72 have been transformed to precedents models and have been included to the knowledge base of the ES.

\section{EXPERT SYSTEM}

The adaptation of MasWIQA to the decision of expert system tasks in collision avoidance situations is presented in Fig. 7. This "Block and line" view is chosen specially, so that it is corresponded to the typical scheme of the ES. In Fig. 7 the structure of the ES is presented against background of the QA-model to emphasize functional character of immersing the ES to the data model of the QA-type. 


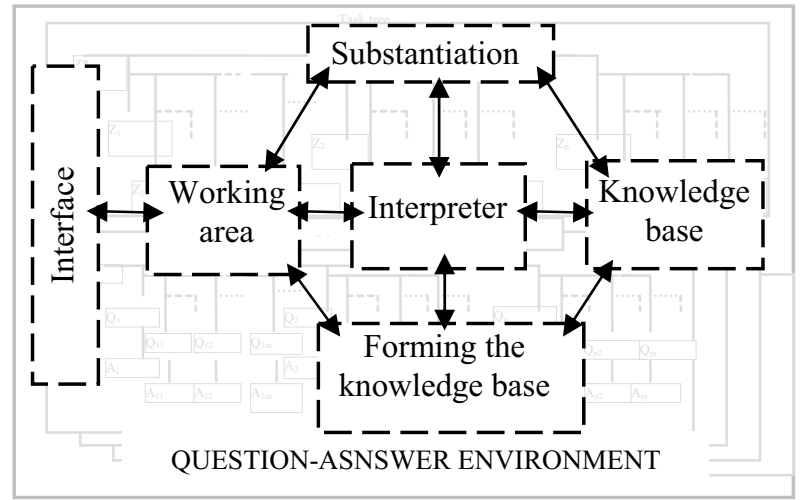

Figure 7. Question Answer Expert system

Any function of the ES is implemented as solving the corresponding task included to the tasks tree. Therefore with any task connects its QA-protocol the creating and using of which is similar the work with the program but the program of the QA-type (Sosnin 2008). WIQA includes the interpreter of the QA-program the execution of which plays the role of the model of reasoning. So any function of the ES is implemented uniformly as modeling of reasoning the agent or user correspondingly.

The human interaction with the ES for choosing the appropriate precedent is fulfilling by two steps. At the first step the list of keys and rating of any precedent are used for roughly choosing. At the second step the work with QA-protocols and predicate models of precedents are used for increasing the adequateness of the choice.

Two steps of the access of the user to the knowledge base is a general technique. In real practice the agent of the own vessel fulfils the work of the first step automatically and periodically. Two steps are useful for checking the results of the choice and in the task of training.

The interaction of any agent with the ES is being implemented similarly the automatic "reasoning" aimed on checking the conditions of appropriate precedents. All chosen precedents for agents register in QAprotocols so that the predictable behavior of agents are accessible for the person who is responsible for the collision avoidance. It is necessary to mention that the interaction of agents with the ES fulfils periodically on the base of the real time data.

The access to the knowledge base is not single cause for the interaction of the user with the ES. The user has the direct access to any task of the tasks tree and therefore to any QA-protocol (or QA-program, or QA-model) in any its state. He can use such access for analysis of solution processes in any interval of time and for modeling the evolving of collision avoidance events.

The interacting of the user with agents is especially important. Such interaction is implemented in two forms. Agents are initiators of the first version of real time interacting. When any agent in its interaction with the ES discovers the danger situation with the vessel $V_{0}$ the agent informs the user about it.
The initiator of the second version of the interaction with agents is the user. The information from agents about their actual relations with the COLREG gives the user the possibility to compute a set of potential facts about changing the vessels movement in the chosen interval of time.

\section{SIMULATION OF PRECEDENTS}

The precedents in accordance with their types and structures are filled by the informational content extracted from the COLREG-72 and from informational sources about "a good sea practice".

The kernel of the knowledge base includes 145 rules (precedents) each of which corresponds of the definite COLREG rule or its detailed version. The content of precedents will be demonstrated on the example of "Rule 15" which regulates the normative behavior in situations of crossing for power-driven vessels $\mathrm{V}^{\mathrm{PD}}{ }_{1}$ and $\mathrm{V}^{\mathrm{PD}}$.

The textual model of the presented precedent (production) repeats the text of the corresponding rule "When two power-driven vessels $V_{-} 1$ and V_2 are crossing so as to involve risk of collision, the vessel...". The predicate model presents the content of the production in the following form:

$\operatorname{Has}_{1}(V$ 1, VPD1)\&Has1(V_2, VPD2)\&

$\& \operatorname{Has} 2(V$ 1,place 1)\&Has2(Vs-2, place 2) \&

$\& \operatorname{Has} 3\left(V_{-}\right.$1, bear_1)\&Has3(V_2, bear_2) \& \&

Has4 $(\bar{V}$ 1, vel_1) \& Has4 $(\bar{V} 2$, vel_ $)$ \&

$\& \operatorname{Has} \overline{5}\left(V_{1} 1\right.$, "keep out of the way") \& \& (bear $1-$ bear_2 $\left.>11,5^{\circ}\right) \&$

$\&\left(\mathrm{CPA}-\mathrm{D}^{\mathrm{DA}}-\Delta \mathrm{D}_{1} \leq 0\right) \rightarrow$ Manoeuvre $M_{i}$, where number " $11,5^{\circ}$ " is a number from the "good sea practice" and "manoeuvre Mi" is a version of the necessary behavior of the vessel V_1.

The QA-model (protocol) of the precedent presents the dialog aimed at the logical estimation of the condition part of the predicate model. The source code is formed as a result of pre-translating the predicate model on the C\# code which is compiled to the executable code.

simulation is aimed at the estimation of collision threats for own vessel $\mathrm{V}_{0}$ with others moving vessels. The estimation includes two line of the behavior for the sailor on duty and for agents modeling vessels.

The sailor on duty must act in accordance with COLREG'72 but this document is a very complicated. Therefore all rules of COLREG' 72 have been

\section{CONCLUSION}

The paper presents the multi-agent approach to the solution of the data fusion task which is implemented on the base of question-answer means.

The suggestions are based on the usage of four classes of agents. The first class of agents is intended for the work with primary information about characteristics of the dynamic objects received from several RSs and equipments of other types. Agents of this class provide transforming the primary informational flows to the 
normative forms. Any agent of the second class is responsible for processing the redundant information about definite characteristic from its normalized flows which correspond to the acted RSs. Agents of the third class are solving the data association task and the task of grouping the targets. The solution process of the data fusion task is a continuous work in which the targets visualization supports by agents of the fourth class. Vessels agents included in the fourth class take part in solving the collision avoidance task.

There are a number of positive effects which are achieved in data fusion and in the collision avoidance practice by the usage of agents in the base of the QAapproach and means of MasWIQA:

1. The QA-approach supports the uniform way of modeling any task which is being solved for monitoring the nearby surrounding of the own vessel.

2. QA-means give the possibility for modeling any agent as the specialized task with subordinated subtasks in the tasks tree of the monitoring system MasWIQA.

3. The multi-agent implementation promotes the parallel processing of the informational flows and solving the other presented tasks.

4. Including the multi-agents model to the collision avoidance analysis gives the user the possibility to compute a set of potential facts about changing the vessels movement in chosen interval of time.

5. Uniform QA-means are being used for (preliminary and/or real time) programming and reprogramming any function of MasWIQA and for including the function as the task in the tasks tree in any time.

In this time the described multi-agent system are being investigated and fitted for its developing till the version which could be used in the real sea practice. The main difference between exploitation version and version for training are only in different sources of the input information.

\section{REFERENCES}

Bar-Shalom Y.; T. Kirubarajan; and C. Gokberk. 2005. "Tracking with Classification-Aided Multiframe Data Association" // IEEE Transactions on AES, Vol. 41, No. 3 (Jul), 868-878.

Capponi A. 2004. "Polynomial Time Algorithm for Data Association Problem in Multitarget Tracking" // IEEE Transactions on AES, Vol. 40, No. 4 (Oct), 1398-1410.

Chung Y.; P. Chou; M. Yang; and H. Chen. 2007. "Multiple-Target Tracking with Competitive Hopfield Neural Network Based Data
Association" // IEEE Transactions on AES, Vol. 43, No. 3 (Jul), 1180-1188.

Cockcroft A.N. 2003. "Guide to the Collision Avoidance Rules: International Regulations for Preventing Collisions at Sea", ButterworthHeinemann.

Grabowski M. and W.A. Wallace. 1993. "An Expert System for Maritime Pilots: Its Design and Assessment Using Gaming", Management science, Vol. 39, No. 12 (Dec), 1506-1520.

Kaplan L.M.; Y. Bar-Shalom; and W.D. Blair. 2008. "Assignment Costs for Multiple Sensor Track-toTrack Association" // IEEE Transactions on AES, Vol. 44, No. 2 (Apr), 655-677.

Liu Y.; X. Du; and S. Yang. 2006. "The Design of a Fuzzy-Neural Network for Ship Collision Avoidance", Lecture Notes in Computer Science, vol. 3930, 804-812.

Liu Y. and Ch.Yang. Y. Y. 2007. "A Multiagent-Based Simulation System for Ship Collision Avoidance", International Conference on Intelligent Computing, August, Qingdao, China, 225-256.

Liu Y.; Ch. Yang; and X. Du. 2008. "A CBR-Based Approach for Ship Collision Avoidance", New Frontiers in Applied Artificial Intelligence, Springer Berlin / Heidelberg, 687-697.

Sosnin P. I. 2008. "Question-Answer Models of Decision-Making Tasks in Automated Designing", 22nd, European Conference on Modeling and Simulation, Nicosia, Cyprus, 173-180.

Sosnin P. 2009. "Means of question-answer interaction for collaborative development activity" // Hindawi Publishing Corporation, Advances in Human-Computer Interaction, Vol. 2009, Article ID 619405.

Puranik S. and J. Tugnait. 2007. "Tracking of Multiple Maneuvering Targets using Multiscan JPDA and IMM Filtering" // IEEE Transactions on AES, Vol. 43, No. 1 (Jan), 23-35.

Tran T.; C.J. Harris; and P.A. Wilson. 2000. "Marine collision avoidance expert system", Intelligent Systems \& Control, Honolulu, USA, 27-32.

Waard H.W. 2008. "A new approach to distributed data fusion," Thesis. Available at http://dare.uva.nl/ record/ 288327.

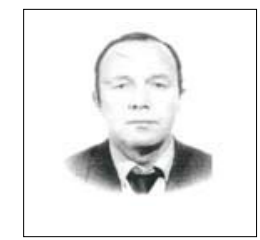

PETR SOSNIN was born in Ulyanovsk in the USSR, on July 12,1945 . He graduated from the Ulyanovsk Polytechnic Institute (1968).

His employment experience includes the Ulyanovsk Polytechnic Institute and Ulyanovsk State Technical University. His special field of interests includes AI applications for the computer aided design. P. Sosnin has defended the doctor degree in Moscow Aviation Institute (1994). He is an author of eight books and more two hundred articles. 